\section{BATHS FOR BURNS}

Davis Forster, M.D., New Smyrna, Fla.

Resident Surgeon, Forster Sanatorium

REPORT OF CASE

A boy, aged 16, burned in an explosion on a gasoline launch and having come through fire to make his escape, was brought by automobile 50 miles, wrapped in a sheet, to the hospital, and was admitted at midnight, July 13, 1919. He was burned on the brow, both ears, the face, nose, mouth, neck, left forearm and hand, right arm, forearm and hand, including the axilla and across the back, down to and on the buttocks and posterior surfaces of the thighs, abdomen and the anterior surfaces of the thighs to the knees. It will be seen that the only uninjured parts by which the patient could be handled were his right arm, both legs below the knee, and his hair, of which he had a large mop. He was well saturated with a hypnotic, and when aroused was delirious.

$\mathrm{He}$ was cleansed with surgical solution of chlorinated soda (Dakin's solution), wrapped in packs of this constantly renewed, and after finally being dried, was coated with paraffin applied with a cotton mop. Parts were covered with large meshed paraffin gauze, which: was found unsuccessful, as the constant rolling of the patient caused the stiff waxladen gauze to become detached. Therefore a light meshed dressing gauze was substituted with better results. The patient was dressed daily with the paraffin, encouraged to drink water freely, was kept on a strictly milk diet, administered every three hours, and had one-fourth grain of morphin sulphate as his condition required.

He voided scantily at twelve hour intervals, the urine being of a syrupy consistency. The bowels were moved by enema. He gradually became more and more septic, the stench being overwhelming. Green flies gathered on the screen outside of his window. The temperature went to 102 , pulse 146 , respiration 39.

On the fifth day, acting on a suggestion for hot boric acid fomentations, ${ }^{1}$ we decided to place the patient in a bathtub full of warm 2 per cent. boric acid solution, which we maintained at a temperature of between 90 and $100 \mathrm{~F}$. warming to the patient's desire and cooling when he seemed exhausted.

He remained in the bath one hour, and on being removed, voided 16 ounces of urine. We felt that he had absorbed a large amount of fluid through his denuded tissue and that the flushing of the kidneys was a happy accompaniment of the mechanical cleansing. When he was taken from the solution, the bleeding parts, particularly the buttocks, were coated with an ointment having a petroleum base, while the rest was treated with paraffin after drying. If the surface is not dried, the application is very painful.

The results of the immersion were so favorable that the next day we kept him in the solution three hours, detaching dead skin and large sloughs. The baths continued daily, changing from boric acid solution to physiologic sodium chlorid solution. The sloughing on the right side of the neck was so extensive that it was feared that a permanent contracture would hold the head in the one-sided position the patient constantly maintained; but gradually the condition improved.

It was remarkable to see the skin come in over the raw surfaces, growing as much as an inch in a day to cover a surface as large as two outspread hands. The immersion assisted in detaching adherent particles of dressing and débris, thus avoiding the rubbing which would have detached tender growths of epithelium.

At the end of three weeks the patient was on his feet, walking in the sunshine, and in four weeks was discharged "rough healed" with only the right ear unhealed, which had an abscess of the cartilaginous structures.

We have since treated a less severe case with as satisfactory results.

1. New Horison Collection of Medical and Surgical Therapy, issued under the Surgeon-Generals of the United States and the British Armies $5: 780$.
ACCIDENTAL PERFORATION OF THE LTERUS DURING CURETTAGE, WITH LACERATION OF THE SMALL INTESTINE

REPORT OF CASE WITH OPERATION AND RECOVERY

John T. Williams, M.D., Boston

Assistant Visiting Surgeon, Boston City Hospital; Assistant in Gynecology, Medical School of Harvard University

Accidental perforation of the uterus during curettage is undoubtedly of frequent occurrence, but for obvious reasons it is seldom reported. It may happen at the hands of a skilful surgeon, and if proper asepsis has been maintained, and the perforation is recognized immediately, harm seldom results. If, however, the perforation is not recognized as soon as it is made, the operator may continue to curet through the perforation, bringing down a loop of intestine (usually ileum), and, as in the case to be reported, severing the bowel from its mesentery before identifying it.

\section{REPORT OF CASE}

Mrs. R., aged 27, had had several previous miscarriages. The Wassermann reaction was +++ . June 29,1919 , being about three and a half months pregnant, she started to miscarry. She was curetted at her home by a local physician, who perforated the uterus, drew down a loop of small intestine, severed it from its mesentery, and tore it completely across before he recognized what he had done.

The patient was admitted about one hour later to the gynecologic service of the Boston City Hospital, where I saw her. She was in good condition, not bleeding, but had several inches of lacerated intestine protruding from the vulva. The abdomen was immediately opened, and the conditions found were these: The ileum was completely torn across about 12 inches from the cecum. The proximal portion had been separated from its mesentery for a distance of 12 inches and passed down through a ragged opening about 1 inch in length in the fundus uteri near the right cornu. The free edge of the mesentery was tacerated, ragged and bruised in appearance. There was a small amount of bloody fluid free in the peritoneal cavity, but no feces.

The loop of ileum that had been separated from its mesentery was resected, the part that passed through the perforation being tied off and withdrawn from below to avoid contamination of the peritoneum by dragging up through the abdomen the portion that had projected from the vulva. The lacerated distal end of the bowel and the bruised margin of the mesentery were trimmed away to get clean-cut tissues to bring together. An end-to-end anastomosis was done, the Connell suture of catgut, reinforced by a continuous suture of Pagenstecher to the peritoneum, being used. The edges of the rent in the uterus wcre trimmed away into clean tissue, and the perforation was closed with interrupted catgut (one layer). A perforation in the distal portion of the ileum about the size of a pinhead was found and closed by a pursestring suture of Pagenstecher. The peritoneum was carefully cleaned by gentle mopping with moist sponges. A cigaret wick was passed to the pelvis. The abdomen was closed.

As the absence of bleeding was taken to indicate that the uterus was empty, nothing was done to the uterine cavity, and the patient was quickly put to bed and the MurphyFowler treatment instituted. She made an uninterrupted recovery. The drain was shortened on the fourth, fifth and sixth days, and was entirely removed on the seventh. There was no drainage from the wound except serum, and the patient left the hospital on the eighteenth day, well except for a granulating sinus at the site of drainage one-half inch in depth.

483 Beacon Street.

Public Health Nursing.--Although a visiting nurse began work in Liverpool sixty years ago, as late as 1901 there were only fifty-three visiting nursing associations and 136 visiting nurses in this country. In 1915 there were 2,066 associations and 4,893 visiting nurses.-Bulletin, State Board of Health of Rhode Island, August, 1919. 